\title{
Efeito preventivo da fisioterapia na redução da incidência de quedas em pacientes com Doença de Alzheimer
}

\author{
Preventive effect of the physical therapy to minimize the occurrence of falls in patients with \\ Alzheimer's Disease
}

\section{Tetsadê CB Piermartiri ${ }^{1}$, Naaraí Camboim Bezerra² ${ }^{2}$ Alexandre Ademar Hoeller ${ }^{3}$}

\begin{abstract}
RESUMO
Esta revisão tem como meta proporcionar melhor compreensão do efeito preventivo da intervenção fisioterapêutica para minimizar a ocorrência de quedas em pacientes com Doença de Alzheimer. A Doença de Alzheimer é a principal causa de demência entre pessoas idosas, apresentando nos últimos anos um crescimento rápido do número de acometidos devido ao aumento da expectativa de vida. Embora o paciente portador de Alzheimer apresente em sua fase inicial apenas perdas cognitivas e de linguagem, a evolução progressiva da doença é capaz de gerar um aumento no déficit de equilíbrio, distúrbios na marcha e a possibilidades de ocorrência de quedas. Apesar de alguns estudos apresentarem dados que comprovem o beneficio da fisioterapia em diminuir o risco de quedas, não foi possível chegar a uma conclusão efetiva sobre o efeito preventivo de quedas através da aplicação desta modalidade terapêutica. A pequena quantidade de estudos que enfatizem o tratamento fisioterapêutico sobre a DA, assim como, a padronização de um método fisioterapêutico no tratamento desta doença, faz com que seja necessário o desenvolvimento de novos estudos e diferentes estratégias de prevenção de quedas no tratamento deste grupo de pacientes, almejando minimizar danos motores e prolongar a independência desses pacientes.
\end{abstract}

Unitermos: Acidente por Quedas. Demência. Doença de Alzheimer. Fisioterapia. Idosos. Prevenção de Acidentes.

Citação: Piermartiri TCB, Bezerra NC, Hoeller AA. Efeito preventivo da fisioterapia na redução da incidência de quedas em pacientes com Doença de Alzheimer.

Trabalho realizado na Universidade Federal de Santa Catarina.

1. Fisioterapeuta, Especialista em Fisioterapia Neuro-funcional, Mestranda em Neurociências da Universidade Federal de Santa Catarina (UFSC).

2. Acadêmica do curso de Medicina, Universidade Federal de Santa Catarina (UFSC).

3. Fisioterapeuta, Mestrando em Neurociências da Universidade Federal de Santa Catarina (UFSC).

\section{SUMMARY}

The purpose of this review is to provide better understanding of the preventive effect resulted by the Physical Therapy intervention to minimize the occurrence of falls in patients with Alzheimer Disease. The Alzheimer Disease is the main cause of dementia between aged people. In recent years it has been showing an increase in the number of patients proportional to the increase of life expectancy. Although the patient carrying Alzheimer presents in its initial phase only cognitive and language losses, the clinical evolution of the disease may increase deficit of balance, gait disturb and the possibilities of falls. Although some works had show data about the beneficial results occasioned by physical therapy treatment decreasing the risks of falls, was not possible obtain a effective conclusion about the preventive effect related to falls through this therapeutic modality. The few number of studies that focus on physical therapy treatment related to $A D$, and the absence of a standard therapeutic method o the treatment of this disease, makes necessity the development of new studies and different strategies of prevention of falls in this group of patients are necessary to minimize motor damages and to draw out the independence of the patients.

Keywords: Accidental Falls. Dementia. Alzheimer Disease. Physical Therapy. Aged. Accident Prevention.

Citation: Piermartiri TCB, Bezerra NC, Hoeller AA. Preventive effect of the physical therapy to minimize the occurrence of falls in patients with Alzheimer's Disease.

Endereço para Correspondência: Tetsadê CB Piermartiri Universidade Federal de Santa Catarina Centro de Ciências Biológicas (CCB) 88040-900 Florianópolis, SC Fone: (48) 3721-5046 e-mail: tetsade@yahoo.com.br

Recebido em: 03/03/08 Revisado em: 04/03/08 a 31/07/08 Aceito em: 01/08/08 Conflito de interesses: não 


\section{INTRODUÇÃO}

A alta incidência de quedas constitui um grande problema de saúde entre os indivíduos de idade avançada, sendo que a freqüência e os danos resultantes destes acidentes tornam-se maiores em longevos acometidos pela Doença de Alzheimer (DA) quando comparados a idosos que não apresentam comprometimentos cognitivos ${ }^{1}$.

A DA é a principal causa de demência entre pessoas idosas, apresentando um crescimento rápido do número de acometidos devido ao aumento da expectativa de vida nos países desenvolvidos e em desenvolvimento. Atualmente, a patologia afeta mais de 4,5 milhões de pessoas nos Estados Unidos da América. No Brasil, o aumento da população idosa será da ordem de 15 vezes entre 1950 e 2025, enquanto estima-se que o aumento populacional como um todo será da ordem de 5 vezes. Estudo epidemiológico realizado na cidade de Catanduva, São Paulo, revelou que dos 1.660 idosos avaliados, $118(7,1 \%)$ apresentaram diagnóstico de demência, sendo 54,1\% diagnosticados com a doença de Alzheimer, 9,3\% demência vascular e $14,4 \%$ doença de Alzheimer associada à demência vascular. Clinicamente, a enfermidade é caracterizada por um declínio gradual e progressivo na memória e em outros domínios de cognição, assim como pela ocorrência freqüente de sintomas comportamentais não cognitivos ${ }^{2-4}$.

Vários estudos demonstram que as quedas ocorridas em idosos trazem sérias conseqüências físicas, psicológicas e sociais, o que reforça a necessidade de prevenção destes acidentes, garantindo ao idoso uma melhor qualidade de vida, autonomia e independência. Sendo assim, o objetivo deste trabalho foi avaliar através de dados literários o efeito preventivo da intervenção fisioterapêutica com intuito de minimizar ocorrências de quedas em pacientes com DA.

\section{MÉTODO}

Foi realizada uma pesquisa bibliográfica empregando as palavras-chaves: Acidente por quedas, Demência, Doença de Alzheimer, Fisioterapia e Prevenção. Essas podiam estar associadas ou não e, quando necessário, complementadas por termos específicos. Os estudos foram identificados em bancos de dados informatizados (Pubmed, Bireme e SciELO). Os critérios de inclusão foram: artigos nos idiomas português, inglês, espanhol ou francês, datados entre os anos 1997 a 2007; artigos relacionados com etiologia, prevalência, aspectos histopatológicos e genéticos presente na demência e doença de Alzheimer; incidência de quedas nos pacientes diagnosticados com demência e doença de Alzheimer; inclusão de abordagens fisioterapêuticas entre os tratamentos utilizados; estudos de casos, artigos originais, estudos randomizados, revisões bibliográficas, sistemáticas e meta-análises foram inclusos.

Apartir dos critérios estabelecidos foram inclusos 57 artigos. Os demais estudos considerados irrelevantes e resumos não foram considerados neste trabalho.

\section{RESULTADOS}

\section{A doença de Alzheimer}

A DA constitui a mais freqüente forma de demência entre idosos nos países industrializados e têm aumentado significantemente nos últimos 50 anos $^{5}$. A estimativa é que $10 \%$ dos idosos americanos com 65 anos e 50\% com 85 anos desenvolvam essa doença. A DA é atualmente a terceira doença mais cara tratada nos Estados Unidos da América, custando aproximadamente 100 bilhões de dólares anuais para a sociedade 6 .

A maior parte dos casos de DA (90\% a $95 \%)$ apresenta início tardio e está relacionada a diversos fatores de risco, como o avanço da idade, gênero feminino, baixo nível educacional, tabagismo, polimorfismo da apolipoproteína E (ApoE) e doenças graves, como diabetes mellitus, hipertensãoe hipercolesterolemia7,8.

As marcas histopatológicas da DA são caracterizadas pela presença de placas senis (ou neuríticas), emaranhados neurofibrilares e perda difusa de neurônios ${ }^{9}$. As placas senis resultam do metabolismo anormal da proteína precursora do amilóide (APP), conduzindo à formação de agregados do peptídeo $\beta$-amilóide ${ }^{10}$. Os emaranhados neurofibrilares formam-se a partir do colapso do citoesqueleto neuronal, decorrente da hiperfosforilação da proteína tau ${ }^{11}$. Além disso, foram identificadas perdas neuronais no córtex entorrinal, hipocampo e córtex frontal, parietal e temporal ${ }^{9}$.

Embora a maioria dos casos de DA ocorra esporadicamente, a desordem é herdada por um padrão dominante autossômico em uma pequena fração de pacientes (menor que $1 \%)^{12}$. Quatro genes têm sido associados com o desenvolvimento da doença, sendo que mutações nos genes codifi- 
cadores para a APP, presenilina 1 (PSEN1) e presenilina 2 (PSEN2) pertencem à DA de início precoce (anterior aos 65 anos) $)^{13-15}$, enquanto o gene da ApoE (apolipoproteína E) encontra-se associado com a forma mais comum da DA de ínício tardio (após 65 anos) ${ }^{16}$.

A doença apresenta uma duração média de 8 a 10 anos. Por motivos desconhecidos, alguns pacientes com Alzheimer apresentam um declínio gradual da função, enquanto outros prosseguem sem deterioração importante ${ }^{17,18}$. Normalmente observam-se prejuízos na marcha e equilíbrio dos indivíduos acometidos, embora em menor grau quando relacionados a outras demências ${ }^{19,20}$.

De acordo com Manchoundia et al., características espaço-temporais de movimentos relacionadas com orientação e velocidade durante a atividade de sentar-se e levantar-se diferem significantemente entre indivíduos com DA e o grupo controle. Entretanto, sujeitos saudáveis (assim como em jovens) mantêm esta característica espaço-temporal quando realizam este tipo de comportamento, sugerindo uma alteração do planejamento motor ou dos processos motores de alto nível devido à $\mathrm{DA}^{21}$.

Atualmente, o tratamento farmacológico considerado padrão para a demência da doença de Alzheimer inclui os inibidores da acetilcolinesterase, para as fases de demência leve a moderada, e a memantina, para as fases moderada à grave ${ }^{22,23}$.

O tratamento fisioterapêutico consiste em programa de exercícios de resistência e fortalecimento que são importantes para aumentar e manter a força muscular assim como melhorar o metabolismo. Exercícios de alongamento são capazes de auxiliar na manutenção ou ganho de flexibilidade. Além destes, atividades relacionadas com o equilíbrio e treino de marcha apresentam papel importante na prevenção de quedas ${ }^{24-26}$.
Risco de quedas na Doença de Alzheimer

A presença de demência se mostrou um fator de risco importante para a ocorrência de quedas em diversos estudos (Tabela 1). Pessoas com DA estão mais propensas a quedas ${ }^{27}$, estando consequentemente mais sujeitas a fraturas ${ }^{31}$ quando comparadas a idosos sem prejuízo cognitivo.

Múltiplos fatores podem estar relacionados ao risco de queda em pacientes com DA, podendo associar-se ao declínio de equilíbrio com prejuízo na marcha e instabilidade postural, uso de medicamentos para o controle de alterações comportamentais e falta de percepção dos comportamentos de risco 32,33 .

Para Camicioli et al., o prejuízo motor está associado ao aumento de quedas em pacientes com $\mathrm{DA}^{34}$. O aumento do comprimento do passo em todas as velocidades de marcha em pacientes com Alzheimer aumenta a incidência de quedas, quando comparado a um grupo de indivíduos saudáveis ${ }^{35}$.

Kato et al. observaram diferenças no desempenho do equilíbrio dos idosos com DA mais acentuada na fase moderada da doença, assim como um declínio da capacidade funcional, progressivo e proporcional à fase da doença ${ }^{36}$.

Horikawa et al. demonstraram que a lesão na substância branca identificada pela ressonância magnética causa distúrbios na manutenção do equilíbrio e aumenta o risco de quedas na DA, sendo que o uso de drogas neuroepiléticas comumente utilizadas no tratamento comportamental e de sintomas psiquiátricos também estão relacionadas com o aumento de quedas, não afetando, no entanto, o equilíbrio ${ }^{37}$.

Fisioterapia e estratégias de prevenção de quedas

Para que haja um bom controle postural, é necessário o correto posicionamento dos mem-

Tabela 1. Presença de demência como um fator de risco para quedas.

\begin{tabular}{|l|c|c|l|}
\hline Autores & (n) & Tipo de estudo & Principais achados \\
\hline Carvalho \& Coutinho, 2002 ${ }^{28}$ & 404 idosos & Caso-controle & $\begin{array}{l}\text { Acidentaram-se dentro de casa 78\% dos idosos com demência, contra 55\% } \\
\text { daqueles sem essa doença. }\end{array}$ \\
\hline Van Doorm et al., 200327 & 59 idosos & Coorteprospectivo & $\begin{array}{l}\text { Pacientes com demência em asilos sofrem 4.05 quedas por ano, comparada } \\
\text { com 2.33 quedas por anos dos idosos sem demência ( }<<0,0001) .\end{array}$ \\
\hline Weller et al., 2004 & 1513 idosos & Prospectivo & $\begin{array}{l}\text { Pacientes com Doença de Alzheimer sofrem mais quedas (46\%) que outros } \\
(35 \%), \text { porém a relação com ocorrência de fraturas é independente da doença. }\end{array}$ \\
\hline Christofoletti et al., 200629 & 20 idosos & Transversal & $\begin{array}{l}\text { Maior risco de quedas no grupo com Doença de Alzheimer, seguido pelos } \\
\text { grupos com Doença de Parkinson e controle. }\end{array}$ \\
\hline Lorbach et al., 200730 & 42 idosos & Caso-controle & $\begin{array}{l}\text { Os pacientes com Doença de Alzheimer possuem significantes riscos de que- } \\
\text { das ( } \mathrm{p}<0,02) \text { comparados com um grupo controle }\end{array}$ \\
\hline
\end{tabular}


bros do corpo em relação ao meio externo. A execução postural pode ser regulada por dois mecanismos: (1) as respostas do feedforward resultam de adaptação postural integrada ao programa motor em altos níveis do controle motor (gânglios basais, córtex cerebral, e cerebelo); (2) as rápidas respostas de feedback provenientes de informações visuais periférica, vestibulares e somato-sensorial são geradas em uns níveis mais baixos do controle motor representados pelo tronco encefálico e pela medula espinhal concernentes com a execução; estas respostas permitem os ajustes posturais necessários para corrigir os distúrbios inesperados que ocorrem possivelmente durante a execução do movimento ${ }^{38}$.

Com o processo biológico do envelhecimento, esses sistemas são afetados e várias etapas do controle postural podem ser suprimidas, diminuindo a capacidade compensatória do sistema, levando a um aumento da instabilidade postural e risco de quedas ${ }^{39}$.

Vários estudos têm demonstrado que $a$ intervenção não farmacológica pode ser eficaz para diminuir o declínio funcional presente nos pacientes com Alzheimer ${ }^{40,41}$.

Estudos recentes demonstraram que intervenções com programas de exercícios melhoram a função motora e têm sido um importante fator preventivo contra o declínio das atividades de vida diária (AVD's), risco de quedas, distúrbios comportamentais e depressão (problemas comumente encontrados em paciente com DA $)^{42-45}$.

O objetivo do tratamento fisioterapêutico é proporcionar uma maior independência funcional ao paciente, empregando para isso técnicas que visam manter a força, o tônus e a elasticidade muscular, além de exercícios capazes de promover maior destreza e funcionalidade durante a execução da marcha e outras AVDs associadas à vida do idoso. Em conjunto, o uso de órteses pode ser eventualmente indicado, atentando para o fato de que a má utilização desta pode conduzir a quedas suplementares e agravamento das patologias associadas como dores articulares e encurtamento muscular ${ }^{46}$.

Pesquisas preliminares sugerem que a fisioterapia tem um importante papel na prevenção de quedas dos pacientes com $\mathrm{DA}^{47}$. Segundo o estudo de Toulotte et al., o treinamento físico que se caracteriza pelo fortalecimento muscular dos membros com faixas elásticas, treino propriocep- tivo com superfície de diferentes texturas, treino de equilíbrio com obstáculos e aumento de flexibilidade, foi capaz de aumentar significantemente a marcha, mobilidade e flexibilidade, diminuindo consequentemente o número de quedas, auxiliando a manutenção da independência. A ausência de estímulos no grupo controle conduziu ao aumento do número de quedas, assim como na perda de autonomia destes pacientes ${ }^{26}$.

Roland et al. utilizaram um programa de exercícios de resistência adaptado às capacidades individuais dos pacientes com caminhadas e exercícios em bicicleta. Após aproximadamente 7 semanas (5-12 semanas), encontraram uma diminuição significante no risco de quedas e problemas comportamentais dos 23 indivíduos avaliados com DA entre 71 e 92 anos de idade ${ }^{48}$.

Alguns estudos têm demonstrado que os tratamentos multidisciplinares, que incluem o uso de drogas psicotrópicas, exercícios fisioterapêuticos e terapia ocupacional, reduzem significantemente a incidência de quedas em idosos com 65 anos ou mais, que apresentam histórico de quedas $^{49-51}$.

Thomas et al. avaliaram os efeitos causados por exercícios de resistência com o intuito de aumentar a força e a funcionalidade dos membros inferiores de pessoas com demência. A intervenção consistiu no treinamento progressivo de resistência com intensidade moderada dos músculos extensores da coxa, abdutores, extensores e flexores do joelho e dorso-flexores, utilizando para isso faixas de resistência com uma freqüência de 3 vezes por semana durante 6 semanas. Os pacientes apresentaram melhoras em alguns grupos musculares como quadríceps e extensores da coxa, assim como bons resultados em testes de marcha e "sentar-levantar"; no entanto, houve um declínio da força em outros músculos como dorsiflexores e iliopsoas. Os resultados apontam para uma intervenção em potencial de longa duração e de maior intensidade para produzir efeitos benéficos sobre a função neuromuscular de pessoas com demên$\mathrm{cia}^{52}$.

Rebelatto et al. avaliaram recentemente 94 indivíduos com idade média de 60 anos que participaram durante um ano do Programa de Revitalização de Adultos em São Carlos, SP. Os indivíduos realizaram 123 sessões de atividade física que incluíam sessões de alongamentos, exercícios de resistência aeróbica, força, potência e resistência, ati- 
vidades de coordenação, agilidade e flexibilidade, exercícios respiratórios e de relaxamento, sofrendo avaliações periódicas quanto à força de preensão manual, equilíbrio dinâmico e flexibilidade. Como resultado, mulheres e homens experimentaram ganho de flexibilidade e de equilíbrio ao longo do programa porém não reduziram o número de quedas significativamente, havendo melhora da força muscular apenas no gênero feminino. Os indivíduos que relataram terem caído pelo menos uma vez no decorrer do ano ( $28,72 \%$ dos participantes) ganharam força e flexibilidade, porém o equilíbrio dinâmico não melhorou. $\mathrm{O}$ grupo de indivíduos que não caiu apresentou ganho no equilíbrio e na flexibilidade, mas não na força de preensão manual. Como conclusão, obteve-se que após um ano, o número de quedas dos participantes não reduziu de forma significativa. Entre os participantes que caíram pelo menos uma vez ao longo do período estudado, a evolução do número de quedas se deu de maneira inversa à evolução da força e do equilíbrio dinâmico, mas não apresentou relação com a evolução da flexibilidade ${ }^{53}$.

Recentemente, Oliver et al. realizaram uma revisão sistemática e de meta-análise, encontrando evidências de modesta redução na taxa de queda em pacientes hospitalizados que receberam intervenção multidisciplinar ${ }^{54}$. Shaw et al. realizaram um estudo aleatório com 274 idosos com prejuízo cognitivo e demência que se apresentavam ao departamento de demência após a queda. Não foi observada diferença significativa em relação aos idosos saudáveis na diminuição do risco de quedas nos pacientes que receberam intervenção multidisciplinar. $O$ tratamento consistiu na investigação e tratamento médico de outros distúrbios não tratados, revisão e modificação do tratamento farmacológico, além de acompanhamento oftalmológico e cardiológico, supervisão fisioterapêutica com programa de exercícios de 3 meses enfatizando treinamento de marcha, balanço, transferência e mobilidade, fortalecimento e flexibilidade dos membros, além da adaptação da casa do paciente pelo terapeuta ocupacional visando diminuir o número de acidentes domésticos ${ }^{32}$.

Os mesmos autores sugerem que, para que haja uma prevenção efetiva de quedas em pacientes com demência e disfunções cognitivas, são necessárias diferentes estratégias daquelas utilizadas em idosos que não apresentam disfunções cognitivas. Além disso, a utilização de medicamentos psicotrópicos e cardiovasculares tem demonstrado grande relevância como fator de risco para quedas, assim como a hipotensão ortostática, que se apresenta como grande fator de risco para quedas em idosos com demência ${ }^{55,56}$. A partir do exposto, a fisioterapia poderia ser empregada por um período maior, sendo ainda que interações que modificam os fatores de risco cardiovasculares podem ter relevante importância durante o tratamento ${ }^{32,57}$.

\section{CONCLUSÃO}

A incidência de quedas é comum em pacientes acometidos pela Doença de Alzheimer. Identificar os fatores de risco e desenvolver estratégias de prevenção de quedas pode resultar na melhora da manutenção da qualidade de vida destes pacientes.

Apesar de alguns estudos apresentarem dados que comprovem o benefício da fisioterapia em diminuir o risco de quedas, não foi possível chegar a uma conclusão efetiva sobre o efeito preventivo de quedas através da aplicação desta modalidade terapêutica. A pequena quantidade de estudos que enfatizem o tratamento fisioterapêutico sobre a DA, assim como a padronização de um método fisioterapêutico no tratamento desta doença, faz com que seja necessário o desenvolvimento de novos estudos e diferentes estratégias de prevenção de quedas no tratamento deste grupo de pacientes, almejando minimizar danos motores e prolongar a independência desses pacientes.

\section{REFERÊNCIAS}

1.Weller I, Schatzker J. Hip fractures and Alzheimer's disease in elderly institutionalized Canadians. Ann Epidemiol 2004;14:319-24.

2.Grossman H, Bergmann C, Parker S. Dementia: a brief review. Mt Sinai J Med 2006;73:985-92.

3. Herrera Junior E, Caramelli P, Nitrini R. Estudo epidemiológico populacional de demência na cidade de Catanduva - estado de São Paulo - Brasil. Rev Psiq Clin 1998;25:70-3.

4.Caselli RJ, Beach TG, Yaari R, Reiman EM. Alzheimer's disease a century later. J Clin Psychiatry. 2006;67:1784-800.

5.Akwa Y, Allain H, Bentue-Ferrer D, Berr C, Bordet R, Geerts $\mathrm{H}$, et al. Neuroprotection and Neurodegenerative disease. From biology to clinical practice. Alzheimer Dis Assoc Disord 2005;19:226-38.

6.Alzheimer's Association (http://alz.org). EUA: Alzheimer's Association. (última atualização 04/2008; citado em 04/2008). Disponível em: http://alz.org.

7.Cankurtaran M, Yavuz BB, Cankurtaran ES, Halil M, Ulger Z, Ariogul S. Risk factors and type of dementia: Vascular or Alzheimer? Arch Gerontol Geriatr 2007; in press. 
8.Casserly I, Topol E. Convergence of atherosclerosis and Alzheimer's disease: inflammation, cholesterol, and misfolded proteins. Lancet 2004;9415:1139-46.

9.Mattson MP. Pathways towards and away from Alzheimer's disease. Nature 2004;430:631-9.

10.Selkoe DJ, Schenk D. Alzheimer's disease: molecular understanding predicts amyloid-based therapeutics Annu Rev Pharmacol Toxicol 2003;43:545-84.

11. Hamdane M, Delobel P, Sambo AV, Smet C, Bégard S, Violleau $A$, et al. Neurofibrillary degeneration of the Alzheimer-type: an alternate pathway to neuronal apoptosis? Biochem Pharmacol 2003;66:1619-25.

12.Rogaeva E, Kawarai T, George-Hyslop PS. Genetic complexity of Alzheimer's disease: successes and challenges. J Alzheimers Dis 2006;9:381-7.

13.Lleo A, Blesa R,Gendre J, Castellvi M, Pastor P, Queralt R, et al. A novel presenilin 2 gene mutation (D439A) in a patient with early-onset Alzheimer's disease. Neurology 2001;57:1926-8.

14.Matsubara-Tsutsui M, Yasuda M, Yamagata H, Nomura, T, Taguchi T, Kohara K, et al. Molecular evidence of presenilin-1 mutation in familial early onset dementia. Am J Med Genet 2002;114:292-8.

15.Sambamurti K, Greig NH, Lahiri DK. Advances in the cellular and molecular biology of the beta-amyloid protein in Alzheimer's disease. Neuromolecular Med 2002;:1-31.

16.Lambert JC, Araria-Goumidi, L, Myllykangas L, Ellis C, Wang JC, Bullido MJ, et al. Contribution of APOE promoter polymorphisms to Alzheimer's disease risk. Neurology 2002;:59-66.

17.American Psychiatric Association. Diagnostic and statistical manual of mental disorders 4 ed., text revision (DSM-IV-TRTM), 2000, 156-8.

18.Bullock R, Hammond G. Realistic expectations: the management of severe Alzheimer disease. Alzheimer Dis Assoc Disord 2003;17:S80-5.

19.Larson EB, Shadlen MF, Wang L, McCormick WC, Bowen JD, Teri $L$, et al. Survival after initial diagnosis of Alzheimer disease. Ann Intern Med 2004;140:501-9.

20.Allan LM, Ballard CG, Burn DJ, Kenny RA. Prevalence and severity of gait disorders in Alzheimer's and non-Alzheimer's dementias. JAGS 2005;53:1681-7.

21.Manckoundia P, Mourey F, Pfitzenmeyer P, Papaxanthis C. Comparison of motor strategies in sit-to-stand and back-tosit motions between healthy and Alzheimer's disease elderly subjects. Neuroscience 2006;137:385-92.

22.Gandía L, Alvarez RM, Hernández-Guijo JM, González-Rubio JM, de Pascual R, Rojo J, et al Anticholinesterases in the treatment of Alzheimer's disease Rev Neurol 2006;42(8):471-7. 23.Tanovi A, Alfaro V. Glutamate-related excitotoxicity neuroprotection with memantine, an uncompetitive antagonist of NMDA-glutamate receptor, in Alzheimer's disease and vascular dementia. Rev Neurol 2006;42:607-16.

24.Teri L, Gibbons LE, McCurry SM, Logsdon RG, Buchner DM, Barlow WE, et al. Exercise plus behavioral management in patients with Alzheimer disease: a randomized controlled trial. JAMA 2003;290:2015-22.

25.Rolland Y, Pillard F, Klapouszczak A, Reynish E, Thomas D, Andrieu $S$, et al. Exercise program for nursing home residents with Alzheimer's disease: a 1-year randomized, controlled trial. JAGS 2007;55:158-65.

26.Toulotte $C$, Fabre C, Dangremont B, Lensel G, Thévenon A. Effects of physical training on the physical capacity of frail, demented patients with a history of falling: a randomised controlled trial. Age Ageing 2003;32:67-73. 27.van Doorn C, Gruber-Baldini AL, Zimmerman S, Hebel JR, Port $C L$, Baumgarten $M$, et al. Dementia as a Risk Factor for Falls and Fall Injuries Among Nursing Home Residents. J Am Geriatr Soc 2003;51:1213-8.

28.Carvalho AM, Coutinho ESF. Demência como fator de risco para fraturas graves em idosos. Rev Saúde Pub 2002;36:44854.

29.Chistofoletti G, Oliani MM, Gobbi LTB, Gobbi S, Stella F. Risco de quedas em idosos com doença de Parkinson e demência de Alzheimer: um estudo transversal. Rev Bras Fisioter 2006;10:429-33.

30.Lorbach ER, Webster KE, Menz HB, Wittwer JE, Merory JR. Hysiological falls risk assessment in older people with Alzheimer's disease. Dement Geriatr Cogn Disord 2007;24:260-5.

31.Sato Y, Kanoko T, Satoh K, Iwamoto J. Risk factors for hip fracture among elderly patients with Alzheimer's disease. J Neurol Sci 2004;223:107-12.

32.Shaw FE, Bond J, Richardson DA, Dawson P, Steen IN, McKeith IG, et al. Multifactorial intervention after a fall in older people with cognitive impairment and dementia presenting to the accident and emergency department: randomised controlled trial. Br Med J 2003;326:73-7.

33.Chaimowick F, Ferreira TJXM, Miguel DFA. Use of psychoactive drugs and related falls among older people living in a community in Brazil. Rev Saúde Pub 2000;34:631-5.

34.Camicioli R, Licis L. Motor impairment predicts falls in specialized Alzheimer care units. Alzheimer Dis Assoc Disord 2004;18:214-8.

35.Webster KE, Merory JR, Wittwer JE. Gait variability in community dwelling adults with Alzheimer disease. Alzheimer Dis Assoc Disord 2006;20:37-40.

36.Kato EM, Radanovic M, Smid J, Caramelli P, Brucki SM, Nitrini R. Evaluation of the balance control in Alzheimer disease. Arq Neuropsiquiatr 2005;63:S110.

37. Horikawa E, Matsui T, Arai H, Seki T, Iwasaki K, Sasaki H. Risk of Falls in Alzheimer's disease: a prospective study. Internal Med 2005;44:717-21.

38.Lalonde R, Strazielle C. Brain regions and genes affecting postural control. Prog Neurobiol 2007;81:45-60.

39.Sullivan EV, Rose J, Rohlfing T, Pfefferbaum A. Postural sway reduction in aging men and women: Relation to brain structure, cognitive status, and stabilizing factors. Neurobiol Aging 2007; in press.

40.Yu F, Kolanowski AM, Strumpf NE, Eslinger PJ. Improving cognition and function through exercise intervention in Alzheimer's disease. J Nur Scholar 2006;38:358-65.

41.Heyn P, Abreu BC, Ottenbacher KJ. The effects of exercise training on elderly persons with cognitive impairment and dementia: a meta-analysis. Arch Phys Med Rehabil 2004;85:1694704.

42.Cohen-Mansfield J, Mintzer JE. Time for change: the role of nonpharmacological interventions in treating behavior problems in nursing home residents with dementia. Alzheimer Dis Assoc Disord 2005; 19:37-40.

43.Lautenschlager NT, Almeida OP, Flicker L, Janca A. Can physical activity improve the mental health of older adults? Ann Gen Hosp Psychiatr 2004;3:12.

44.Logsdon, RG, McCurry SM, Teri L. A home health care approach to exercise for person with Alzheimer's Disease. Care Manag 2005;6:90-7.

45.Landi F, Russo A, Bernabei R. Physical activity and behavior in the elderly: a pilot study. Arch Gerontol Geriatr Suppl 2004;9:235-41. 
46.Strubel D, Jacquot JM, Martin-Hunyadi C. Dementia and falls [Article in French]. Ann Readapt Med Phys 2001;44:4-12.

47.Puisieux F, Pardessus V, Bombois S.Dementia and falls: two related syndromes in old age [article in French]. Psychol Neuropsychiatr Vieil 2005;3:271-9.

48. Rolland Y, Rival L, Pillard F, Lafont C, Rivére D, Albaréde J, et al. Feasibily of regular physical exercise for patients with moderate to severe Alzheimer disease. J Nut Health Aging 2000;:109-13.

49.Peeters GM, de Vries OJ, Elders PJ, Pluijm SM, Bouter LM, Lips P. Prevention of fall incidents in patients with a high risk of falling: design of a randomised controlled trial with an economic evaluation of the effect of multidisciplinary transmural care. BMC Geriatr 2007;2:7-15.

50.Robertson MC, Devlin N, Gardner MM, Campbell AJ. Effectiveness and economic evaluation of a nurse delivered homeexercise programme to prevent falls: randomised controlled trial. BMJ 2001;322:697-701.

51.Gillespie LD, Gillespie WJ, Robertson MC, Lambe SE, Cumming RG, Rowe $\mathrm{BH}$. Interventions for preventing falls in elderly people. Cochrane Database Syst Rev 2003;4:CD000340.
52.Thomas VS, Hageman PA. Can neuromuscular strength and function in people with dementia be rehabilitated using resistance-exercise training? Results from a preliminary intervention study. J Gerontol 2003;58A:746-51.

53.Rebelatto JR, Castro AP. Efeito do programa de revitalização de adultos sobre a ocorrência de quedas dos participantes. Rev Bras Fisioter 2007;11:383-9.

54.Oliver D, Connelly JB, Victor CR, Shaw FE, Whitehead A, Genc $Y$, et al. Strategies to prevent falls and fractures in hospitals and care homes and effect of cognitive impairment: systematic review and meta-analyses. Br Med J 2007;334:82-5.

55.Leipzig RM, Cumming RG, Tinetti ME. Drugs and falls in older people: a systematic review and meta-analysis: II. Cardiac and analgesis drugs. JAGS 1999;47:40-50.

56.Passant U, Warkentin S, Gustafson L. Orthostatic hypotension and low blood pressure in organic dementia: a study of prevalence and related clinical characteristics. Int J Geriatr Psychiatr 1997; 12:395-403.

57.Shaw FE. Prevention of falls in older people with dementia J Neural Transm 2007;114:1259-64. 